\title{
covid-19 Keep calm and carry on learning: using Microsoft Teams to deliver a medical education programme during the COVID-19 pandemic
}

\author{
Authors: Daisy Henderson, ${ }^{\mathrm{A}}$ Hannah Woodcock, ${ }^{\mathrm{B}}$ Jay Mehta, ${ }^{\mathrm{C}}$ Nuzhath Khan, ${ }^{\mathrm{D}}$ Victoria Shivji, ${ }^{\mathrm{E}}$ Charlotte Richardson, ${ }^{\mathrm{D}}$ \\ Haleema Aya, ${ }^{F}$ Shier Ziser, ${ }^{F}$ Gabriele Pollara ${ }^{G}$ and Aine Burns ${ }^{H}$
}

The outbreak of COVID-19 in the UK in March 2020 required a radical remodelling of the medical workforce at Royal Free London NHS Foundation Trust to prepare for the anticipated surge of hospital admissions. The provision of relevant teaching and training was immediately identified as a priority, particularly for staff due to work outside their regular medical specialty. Rather than deliver face-to-face teaching, doctors at the Trust utilised Microsoft Teams, an online communications and collaboration platform, to deliver a multi-disciplinary Trust-wide education programme responsive to the needs of surveyed medical staff. To date members of 18 departments across the Trust have delivered 51 virtual teaching sessions which have been viewed 3,814 times. During this pandemic the virtual education programme has facilitated rapid dissemination of new information and provided a platform for discussion and unity amongst colleagues with overwhelmingly positive feedback from both learners and teachers.

KEYWORDS: COVID-19, medical education, teaching

DOI: $10.7861 /$ fhj.2020-0071

Responding to the pandemic and identification of educational needs

In early February 2020, the first patient with confirmed COVID-19 infection was admitted to Royal Free Hospital (RFH) site of the

Authors: ${ }^{A}$ senior clinical teaching fellow, Sheila Sherlock Postgraduate Medical Education Centre, London, UK; ${ }^{B}$ clinical lecturer in respiratory medicine, Royal Free London NHS Foundation Trust, London, UK; ${ }^{c}$ chief medical information officer, Royal Free London NHS Foundation Trust, London, UK; Dinfectious diseases specialist registrar, Royal Free London NHS Foundation Trust, London, UK; Esenior clinical fellow in infectious diseases education and research, Royal Free London NHS Foundation Trust, London, UK; FFoundation year 2 doctor, Sheila Sherlock Postgraduate Medical Education Centre, London, UK; ${ }^{6}$ NIHR clinical lecturer in medical microbiology, Royal Free London NHS Foundation Trust, London, UK and University College London, London, UK; ${ }^{H}$ professor of postgraduate medical education and nephrology, Sheila Sherlock Postgraduate Medical Education Centre, London, UK and Royal Free London NHS Foundation Trust, London, UK
Royal Free London NHS Foundation Trust. Within six weeks the number of confirmed cases reached 50 and the working pattern of medical doctors underwent a radical remodelling in response to the anticipated surge in admissions as the pandemic ensued over the coming weeks.

Within 24 hours, individual medical speciality teams were replaced with one united workforce consisting of 160 junior doctors and consultants to provide 24 -hour cover for acute medical admissions and medical wards, for which COVID-19 was predicted to encompass the bulk of the workload. Specialists in dermatology, haematology, histopathology, immunology, neurology and ophthalmology joined general medical doctors to supplement the workforce on the medical 'super rota'. Workforce numbers were further bolstered by doctors returning to frontline duties after a period of absence and medical student volunteers. Following redeployment, many doctors were now working outside both their field of expertise and their comfort zone. To meet the challenges of this rapid change in the architecture of the workforce, provision of appropriate teaching and training was immediately identified as a priority, and an education faculty was established as part of the COVID-19 Medical Task Force. The objective of the faculty was to rapidly develop and implement an education programme to increase the preparedness of healthcare professionals involved in the COVID-19 response.

\section{Initiation and development of the education programme}

The initial target audience for the education programme was doctors throughout the Trust of all grades and specialities who would be involved in the COVID-19 response. To help design the programme, the teaching faculty identified the teaching and training needs of the junior doctors and consultants on the new rota through a self-assessment questionnaire disseminated by email within 48 hours of the new medical rota commencing. 45 doctors completed the survey. It was evident from the results that there was an urgent need for a diverse education programme (Table 1) and the education faculty rapidly recruited appropriate expert teachers. The teaching topics most frequently requested were managing a cardiac arrest in a patient with COVID-19 (60\%), the COVID-19 care bundle (58\%) and non-invasive ventilation (58\%).

To enable learners to watch teaching sessions remotely and maintain social distancing the education faculty elected for teaching to be delivered primarily using Microsoft Teams 
Table 1. Questionnaire responses indicating educational needs of staff during COVID-19 outbreak Topic

Number of doctors requesting teaching $(\%)$

COVID-19-specific learning

Donning and doffing personal protective equipment (PPE)

Managing a cardiac arrest in a patient with known or suspected COVID-19

Taking and processing a nasopharyngeal swab

Clinical skills and procedures

Arterial line insertion

$25(56)$

Cannulation

Central line insertion

Chest X-ray interpretation

Electrocardiogram interpretation

Intraosseous (IO) access

Lumbar puncture

Non-invasive ventilation

Paracentesis

Pleural aspiration

Venepuncture

\section{Communication skills}

Breaking bad news

Challenging conversations with relatives

Treatment escalation discussions

\section{Knowledge}

Basic life support

Completion of a medical certificate of cause of death

Confirmation of death

COVID-19 care bundle

Documentation in notes

End of life care

Recognition and assessment of a deteriorating patient

Venous thromboembolism assessment

Tota

(Microsoft, Redmond, USA). This is an online communication and collaboration platform which enables users to host virtual meetings, post in groups and send instant messages, and is protected and monitored within the NHS Secure Boundary, a perimeter security project supporting NHS organisations. ${ }^{2}$ Presenters can share audio, video and content such as a Microsoft PowerPoint presentation; and audience members can interact with each other as well as the presenter through both voice and text. An options appraisal between different clinical communication tools had been conducted by the Trust's clinical informatics team in January 2020, with Microsoft Teams emerging as highly desirable due to its ease of use and feature set. Coincidentally NHS Digital then enabled the roll-out of the platform to NHS organisations for free in response to COVID-19. This combination of pre-existing executive approval and absence of short-term cost allowed the education faculty to choose and rapidly implement Microsoft Teams as its teaching platform. ${ }^{2}$

The timetable and instructions on how to access online teaching sessions were publicised at all three sites within the Trust using email, Microsoft Teams, face-to-face announcements during handovers and with posters. Opt-in email lists were created using the marketing platform MailChimp (Rocket Science Group, Atlanta, USA), allowing the faculty to personalise and segment communication by site and staff role. A live calendar of teaching events was created that participants could subscribe to by following a link or scanning a Quick Response (QR) code.

The content of the online teaching programme was reviewed on a weekly basis in order to keep up with the fast pace of new COVID-19 guidelines and research publications. The audience benefited from the enthusiasm and wealth of expertise of the infectious diseases (ID) department, who had been tracking the burgeoning epidemic and managing the small number of patients with COVID-19 in the Trust before the rota redesign. Within the first week of the rota starting, the ID team delivered daily online teaching sessions on the Royal Free Hospital COVID-19 care bundle. This was complemented by additional ID sessions on use of personal protective equipment (PPE), antibiotic prescribing in the context of COVID-19 and an update on the COVID-19 clinical trials taking place in the Trust. In addition, a weekly journal club led by the ID team permitted rapid review of key research publications that shaped the practice of COVID-19, including factors associated with clinical outcomes, novel diagnostics and therapeutics, as well as challenges of community and nosocomial transmission. These and other selected papers published in this fast-moving field were uploaded to a dedicated channel on Microsoft Teams, facilitating asynchronous text discussions relating to these publications.

To further address the questionnaire responses, the online teaching timetable was complemented by small-group multidisciplinary simulation sessions focusing on key areas, such as the assessment of an acutely unwell patient, advanced life support for patients with known or suspected COVID-19 and tracheostomy care, and on clinical and procedural skills training. Social distancing was maintained throughout these sessions.

\section{Progress and response}

To date there have been 51 live virtual teaching sessions which cumulatively have been viewed 3,814 times (mean $=75$ views per session). These statistics do not include learners that viewed teaching sessions together on one device and it is likely that the true number of participants far exceeds this number. From the outset teaching sessions were attended by doctors of all grades. More recently, teaching sessions have also been attended by nursing staff. This is evidenced by feedback questionnaires which were completed by a wide range of responders: $25 \%$ foundation year doctors, $11 \%$ core trainee doctors, $30 \%$ specialist registrar doctors, $31 \%$ consultant grade doctors and $3 \%$ nurses $(n=278)$.

Teaching sessions were scheduled at $1 \mathrm{pm}$ and $4 \mathrm{pm}$ on weekdays during the likely break times for staff on the new medical rota. 
Table 2. Virtual teaching sessions delivered COVID-19 outbreak

\begin{tabular}{|c|c|}
\hline Speciality & Topic \\
\hline Cardiology & $\begin{array}{l}\text { Cardiac complications in COVID-19 } \\
\text { Thrombotic events in COVID-19 }\end{array}$ \\
\hline Diabetes and endocrine & $\begin{array}{l}\text { Addison's disease } \\
\text { Diabetic emergencies } \\
\text { Hyponatraemia } \\
\text { Thyrotoxicosis and thyroid storm } \\
\text { Update in type II diabetes }\end{array}$ \\
\hline Emergency department & Conversation between ED and intensive care unit: learning from the intensive care unit \\
\hline Health services for elderly care (HSEP) & $\begin{array}{l}\text { Delirium and COVID-19 } \\
\text { Frailty and COVID-19 }\end{array}$ \\
\hline Infectious diseases & $\begin{array}{l}\text { Clinical trials } \\
\text { COVID-19 care bundle } \\
\text { Guidance on antibiotic prescribing in COVID-19 } \\
\text { Learning from COVID-19 experience in Hong Kong } \\
\text { Personal protective equipment }\end{array}$ \\
\hline Infectious diseases journal club & $\begin{array}{l}\text { Antibodies in COVID-19: diagnostic and therapeutic uses } \\
\text { A trial of lopinavir-ritonavir in adults hospitalised with severe COVID-19 } \\
\text { Clinical course and risk factors for mortality of adult inpatients with COVID-19 } \\
\text { Exploring COVID-19 mortality in the black, Asian and minority ethnic groups } \\
\text { SARS-CoV-2 viral loads and the assessment of hydroxychloroquine } \\
\text { The challenge of asymptomatic transmission in COVID-19 } \\
\text { The effect of social distancing measures on transmission of SARS-CoV-2 }\end{array}$ \\
\hline Intensive care & $\begin{array}{l}\text { Conversation between ED and intensive care unit: learning from the intensive care unit } \\
\text { Mechanical ventilation } \\
\text { Pulmonary and renal complications in COVID-19 }\end{array}$ \\
\hline Medical education & Wellbeing \\
\hline Medical Examiner Officer & Guidance on completion of a medical certificate of death (MCCD) \\
\hline Ophthalmology & COVID-19 Eye-CU \\
\hline Palliative medicine & $\begin{array}{l}\text { Breaking bad news } \\
\text { Cornea donation } \\
\text { End of life care }\end{array}$ \\
\hline Physiotherapy & Proning in COVID-19 \\
\hline Psychiatry & Resilience during COVID-19 \\
\hline Radiology & $\begin{array}{l}\text { Chest X-ray interpretation in COVID-19 } \\
\text { General CT interpretation }\end{array}$ \\
\hline Renal & $\begin{array}{l}\text { ACEi/ARBs and COVID-19 } \\
\text { COVID-19 and the kidney } \\
\text { Pulmonary and renal complications in COVID-19 }\end{array}$ \\
\hline Respiratory & $\begin{array}{l}\text { Continuous positive airway pressure therapy (CPAP) } \\
\text { COVID-19 respiratory follow-up } \\
\text { Oxygen delivery in COVID-19 }\end{array}$ \\
\hline Rheumatology & COVID-19 and the cytokine storm \\
\hline Senior management team & In conversation with the chief executive \\
\hline
\end{tabular}

Attendance was encouraged by senior staff at the start of every shift and by rota coordinators in weekly email bulletins. This facilitated integration of the teaching programme into the working day. Those not at work were able to join sessions remotely.

Clinicians from 18 specialities have contributed to the programme (Table 2). Respiratory and critical care healthcare professionals provided teaching on oxygen delivery, continuous positive airway pressure therapy and mechanical ventilation. Radiologists delivered sessions covering chest X-ray and computed tomography (CT) scan interpretation skills in COVID-19 patients. As emerging evidence suggested that COVID-19 infection affects multiple organ systems, specialist teaching was delivered on COVID-19-related thrombotic events, cardiology, and endocrine and renal disease. The palliative care team provided sessions on 
end-of-life care and communication skills, addressing a challenge faced by many doctors, namely the frequent need to break bad news and conduct difficult family discussions over the telephone. ${ }^{3}$ Online sessions were held to promote wellbeing and resilience of healthcare workers. All teaching was interactive with, to date, over 400 questions and responses on the chat forum by learners, with a mean of 8.5 questions per session.

After each session the audience was invited to complete an online Likert scale feedback form. ${ }^{4}$ Responses were overwhelmingly positive. $97 \%$ of participants felt being able to access teaching on Microsoft Teams was useful $(n=287)$ and $96 \%$ felt the teaching session was relevant to their practice $(n=285) .99 \%$ found teaching on Microsoft Teams improved staff morale $(n=180)$. Analysis of text responses indicated that staff and students wanted access to the online teaching in their own time and all teaching sessions are now recorded and uploaded to a shared area on Microsoft Teams.

Feedback from teaching session presenters was also positive. 95\% of the presenters felt Microsoft Teams was an effective platform for teaching and $84 \%$ felt they would be likely to use the platform again for teaching purposes $(n=19)$. Presenters felt the advantages of using Microsoft Teams as a platform for virtual teaching were the possibility of remote access to teaching $(n=8)$, the wide reach of the teaching programme $(n=7)$, the ability to maintain social distancing $(n=3)$, and the ability to interact with the audience $(n=3)$.

\section{Learning from the learning}

At a time that could have felt physically and psychologically isolating for healthcare workers at our Trust, we present the first case study of a fully multi-disciplinary, Trust-wide virtual education programme that was responsive to the educational needs of surveyed medical staff during the COVID-19 outbreak in the UK. Previous studies have identified some of the opportunities and challenges that accompany virtual teaching programmes within selected specialties, ${ }^{5-7}$ but feedback from our study revealed the programme to be well received by learners, addressing their educational needs, and equally was felt to be a rewarding and effective form of information delivery by teachers.

Our study has some clear limitations. Teaching sessions were predominantly focused on the educational needs of doctors, who accounted for $97 \%$ of those completing the feedback questionnaires, and thus the suitability of this teaching format for other healthcare professionals cannot be generalised. Our feedback questionnaire focused on the acceptance of teaching sessions and the use of Microsoft Teams as a platform for virtual teaching but did not assess the learning carried out during the sessions, nor how durable the knowledge gained was. Finally, we were not able to evaluate how this method of delivering teaching compares to more traditional face-to-face forms of teaching, an aspect that will need to be considered as the programme evolves following the current
COVID-19 outbreak peak. We recommend that when implementing similar education programmes, staff are invited to complete a follow-up questionnaire in order to ensure the programme is responsive to their changing educational needs and working patterns.

\section{Conclusion}

We demonstrate that despite the challenges placed on healthcare delivery by a pandemic, virtual learning opportunities are accessible, acceptable and informative. This opens up the possibility of expanding virtual teaching in undergraduate and postgraduate education, ${ }^{8}$ and using it in conjunction with traditional face-to-face approaches after the COVID-19 pandemic has passed.

\section{Conflicts of interest}

The authors do not declare any conflicts of interest. This publication is neither affiliated with nor authorised, sponsored or approved by the Microsoft Corporation.

\section{References}

1 Adelaja I, Sayma M, Walton $\mathrm{H}$ et al. A comprehensive hospital agile preparedness (CHAPs) tool for pandemic preparedness, based on the COVID-19 experience. Future Healthc J 2020;7:165-8.

2 NHS Digital. Messaging tool for NHS to support remote working during coronavirus outbreak. https://digital.nhs.uk/news-andevents/latest-news/messaging-tool-for-nhs-to-support-remoteworking-during-coronavirus-outbreak.

3 Wallace CL, Wladkowski SP, Gibson A, White P. Grief during the COVID-19 pandemic: considerations for palliative care providers. J Pain Symptom Manage 2020;60:e70-6.

4 Lyons OTA, Smith C, Winston JS et al. Impact of UK academic foundation programmes on aspirations to pursue a career in academia. Med Educ 2010:44:996-1005.

5 Almarzooq ZI, Lopes M, Kochar A. Virtual learning during the COVID-19 pandemic: a disruptive technology in graduate medical education. J Am Coll Cardiol 2020;75:2635-8.

6 Koumpouras F, Helfgott S. Stand together and deliver: challenges and opportunities for rheumatology education during the COVID19 pandemic. Arthritis Rheumatol 2020;72:1064-6.

7 Chick RC, Clifton GT, Peace KM et al. Using technology to maintain the education of residents during the COVID-19 pandemic. J Surg Educ 2020, in press (doi: 10.1016/j.jsurg.2020.03.018).

8 Mian A, Khan S. Medical education during pandemics: a UK perspective. BMC Med 2020;18:100.

Address for correspondence: Daisy Henderson, Sheila Sherlock Postgraduate Medical Education Centre, Royal Free London Hospital, Pond Street, London NW3 2QG, UK. Email: daisyhenderson@nhs.net 